\title{
Retinal versus extraretinal influences in flash localization during saccadic eye movements in the presence of a visible background
}

\author{
J. K. O'REGAN \\ CNRS, Paris, France
}

\begin{abstract}
Four experiments examined the relative use of retinal and extraretinal information in judging the location of a stimulus flash presented under normal lighting conditions in the temporal vicinity of an eye saccade. Two previous studies done under normal lighting conditions (N. Bischof \& E. Kramer, 1968, and S. Mateeff, 1978) had hypothesized strong use of extraretinal information. The present study reexamined this work and showed that, in fact, two kinds of retinal effects had been neglected in these studies, and that these alone probably suffice to explain the results. The first retinal effect is related to differences between the response of the visual system to foveal and peripheral stimuli, and may be active even in the dark. The second retinal effect is related to the fact that smearing of the retinal image of the background occurs when the eye moves.
\end{abstract}

When a brief stimulus is flashed up in the visual field sometime near the instant an eye saccade takes place, it is seen as displaced with respect to its true spatial position. Such mislocalization effects have been studied extensively by L. Matin and Pearce (1965), Bischof and Kramer (1968), L. Matin, E. Matin, and Pearce (1969), L. Matin, E. Matin, and Pola (1970), Monahan (1972), and Mateeff (1978). The interest in the mislocation paradigm is that it constitutes a way of examining what L. Matin, E. Matin, and Pearce (1969) called the "extraretinal signal".

The extraretinal signal is a theoretical construct that has its origins in Wundt's, Helmholtz's, and James's theories of space perception and was developed more recently by Sherrington (1918), and von Holst and Mittelstaedt (1950). It is designed to explain why our perception of the visual world is stable despite eye movements. The extraretinal signal is assumed to originate from somewhere other than the retina (e.g., from the eye muscles or their command centers in the brain), and to indicate the position of the eyes with respect to the body. By algebraically combining this extraretinal signal with retinal information about the position of an object on the retina, the visual system should be able to calculate the position of the object with respect to the body even when eye movements are made. If the

I would like to particularly thank Noelle Carlin and Ariane Lévy-Schoen, who were very dedicated subjects. I also am deeply grateful to Ariane Lévy-Schoen, Alex Wertheim, Wayne Shebilske, Andrei Goréa, and anonymous reviewers, who made extensive comments on the manuscript. Finally, I warmly thank Leonard Matin for the suggestions he made in reviewing the paper. Please send reprint requests to Kevin O'Regan, Groupe Regard, Laboratoire de Psychologie Expérimentale, Université René Descartes, EPHE, CNRS, 28 rue Serpente, 75005 Paris, France. extraretinal signal were perfectly accurate, that is, if it changed exactly in step with eye saccades, no errors would be made in locating a brief flash presented near the moment of a saccade. The fact that errors are made in locating such a flash is taken to indicate that the extraretinal signal is sluggish, starting to change slightly before the saccade onset and finishing slightly after it (cf. valuable reviews by L. Matin, 1972, 1976, 1982, Shebilske, 1977, and MacKay, 1973).

While most authors studying mislocation effects have assumed that, for horizontal eye movements, the extraretinal signal can be represented as a simple scalar quantity changing with time over approximately the period of the saccade, Bischof and Kramer (1968) proposed that it must actually be a whole coordinate system of signals, one for each retinal location, with each signal having different time characteristics with respect to the saccade. They were forced into this conclusion by an experiment that showed that the errors made in estimating the position of a flash depended not only on the time of stimulation relative to saccade onset, but also on the retinal location the flash impinged on:

Bischof and Kramer's result forces other work on the mislocation problem to be put into doubt. If their finding of a retinal-locus dependence of mislocation errors is replicable, then experiments studying mislocation of flashes must control for the retinal location the flash impinges upon. If this is not done, then the effects found will be the average of disparate effects for the different retinal locations stimulated, and so will depend mainly on the particular (uncontrolled-for) combination of retinal locations that happen to be used. This fact has been ignored by other authors. For example, Mateeff (1978), in an 
experiment done under conditions very similar to Bischof and Kramer's, plots mislocation data as a function of time, but confounds what may be quite different contributions for different retinal positions stimulated by the flash.

The studies of L. Matin and Pearce 1965, L. Matin, E. Matin, and Pearce (1969), L. Matin, E. Matin, and Pola (1970), and Monahan (1972) should also be reconsidered in the light of Bischof and Kramer's result. In those studies, subjects reported whether a test flash that occurred sometime before, during, or after the eye movement lay to the left or the right of a previously seen fixation target. For every moment of occurrence of the flash, Matin et al. determined a retinal point of subjective equality, whose perceived location matched that of the previously viewed fixation target. If Bischof and Kramer are right, then the extraretinal signal determined by Matin et al. is only the signal corresponding to the fixation target, and other signals would have been found if the initially viewed reference point had been different (L. Matin \& Pearce, 1965, did, in fact, refer to such a possibility). Furthermore, there may possibly be a problem in calculating the point of subjective equality by interpolation along ogives constructed from judgments of "left" and "right," since each of these judgments corresponds to a stimulus falling on a retinal location that is not the point of subjective equality, and so is subject to an extraretinal signal different from the one for the point of subjective equality. If there is not a smooth change in extraretinal signal as we go from one retinal location to the next, then the point of subjective equality cannot be deduced from the behavior of nearby retinal points. While a priori it seems unlikely that the extraretinal signal should behave discontinuously as we move from one retinal point to another, Bischof and Kramer did in fact observe what they called "islands" of discontinuity.

Given the importance of Bischof and Kramer's conclusions regarding the notion of extraretinal signal and the interpretation of mislocation data, it seemed vital to try to replicate their retinal-locus effect, and to check whether the "coordinate system" form of the extraretinal signal was the only way of explaining the data. The series of experiments presented here commences with a replication of the Bischof and Kramer experiment and an extension of it to a wider range of retinal locations stimulated by the flash. Three further experiments then investigate the possibility that the effects found could be accounted for in terms of retinal rather than extraretinal mechanisms.

\section{SACCADE EXPERIMENT}

Bischof and Kramer used time of flash occurrence as an independent variable, and disconfounded time and retinal position stimulated by the flash by doing a post hoc analysis of their data. Here a computer is used to track the retina and to project flashes on predetermined retinal positions, independently of the position the eye has reached in the saccade.

\section{Method}

The subject wore photoelectric eye movement recording glasses that used the scleral reflection technique. The glasses were interfaced to a computer that controlled the display as a function of the subject's eye movements. The computer sampled eye position once every $5 \mathrm{msec}$. The response time of the apparatus combination (eye glasses/amplifier/computer) to an eye movement was measured by having the computer move a simulated eye (consisting of a light patch) on the screen. This time was less than $0.5 \mathrm{msec}$ after the most recent sample taken. The subject's head was fixed by a dental bite, so that the computer could measure absolute eye position in space with an accuracy of $1 / 3 \mathrm{deg}$. This accuracy was maintained throughout by continuous recalibration and automatic checking (cf. O'Regan, 1978). The subject sat $44 \mathrm{~cm}$ from the display screen in a dimly lit room. Ambient lighting and brightness of the display were constant across all three subjects (screen about $60 \mathrm{~cd} / \mathrm{m}^{2}$ ).

Two white triangles, $9.2 \mathrm{~cm}(12 \mathrm{deg})$ apart on a horizontal axis near the middle of the screen, constituted fixation marks $A$ and $B$ on the left and right. The triangles had sides of length $1.5 \mathrm{~cm}$ ( $2 \mathrm{deg}$ ). Figure 1 gives a step-by-step account of an individual experimental trial. In the stimulus phase, the subject makes a saccade from fixation mark $A$ to fixation mark B. Sometime near or during the saccade, the computer generates a stimulus flash, consisting of the letter "I." In the response phase, the subject indicates the position where he saw the flash by moving a cursor controlled by a potentiometer knob. The flashed "I" was $3 \mathrm{~mm}$ $(0.4 \mathrm{deg})$ high and $0.5 \mathrm{~mm}(0.07 \mathrm{deg})$ wide. It was displayed for $13 \mu \mathrm{sec}$ and decayed according to the characteristics of the P31 phosphor whose remanence was reduced using a gray filter. Stimulus intensity was the minimum required to be easily detectable at the greatest eccentricity. It was the same (around $100 \mathrm{~cd} / \mathrm{m}^{2}$ ) for all three subjects. Pilot experiments with different values of flash intensity and ambient lighting showed a negligible effect on the pattern of results.

Two parameters were independently varied: the location the flash stimulated on the retina, and the instant at which the flash occurred. The location the flash stimulated on the retina could be any one of seven possible locations: on the fovea, and \pm 2.4 , \pm 4.8 , or $\pm 7.2 \mathrm{deg}$ from the fovea. On two-thirds of the trials, the flash was triggered at one of six instants following saccade onset: $5,10,15,20,25$, or $30 \mathrm{msec}$ after saccade onset. On one-third of the trials, the flash was triggered at time $t$ after the trial was initiated; $t$ was chosen equal to the latency found at the previous trial measured from trial initiation to saccade onset. In this way, given the variability in saccade latency, some flashes occurring just before or just after the saccade were generated in addition to those triggered at chosen moments during the saccade.

The author (K.O.R.) and two other subjects, one of whom (Subject A.L.S.) was partially naive and the other (Subject N.C.) completely naive, participated in the experiment. The subjects performed the experiment in sessions of 252 trials in which each combination of retinal location and flash trigger instant occurred 4 times (for trigger instants dependent on saccade initiation) or 12 times (when trigger instant depended on trial initiation). The order of trials was random. Owing to data loss through calibration error, some subjects sat for more sessions than others (K.O.R., 4 sessions; A.L.S., 5; N.C., 5).

\section{Results}

Figures 3, 4, and 5 plot the data for the three subjects. In each figure, seven separate graphs are plotted, one for each of the seven retinal locations stimulated by the flash. If responses were veridical, they should 


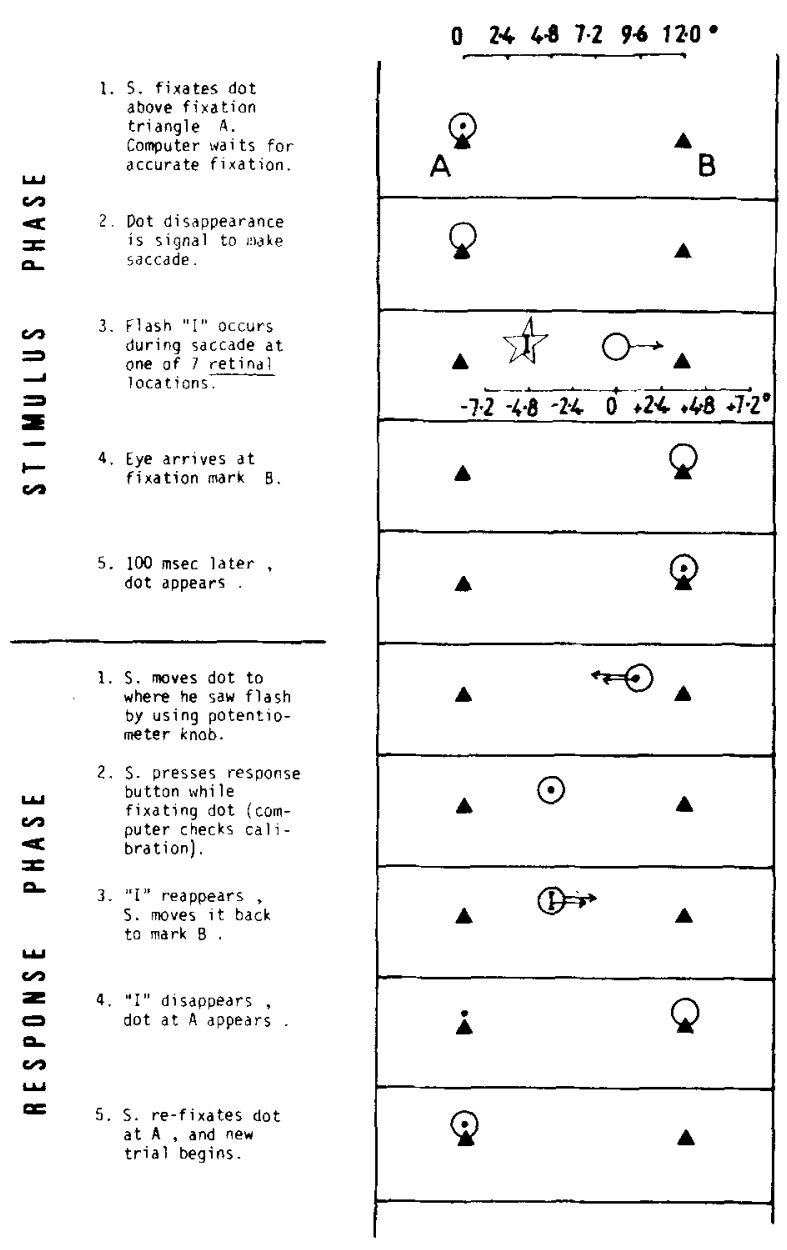

Figure 1. Experimental procedure for the saccade experiment. $A$ and $B$ are white triangles continuously visible on the computer screen. The only things the subject saw on the screen were the fixaton marks $A$ and $B$, and the fiash. The circle and other ballentions in the figure were not visible. Each trial contained a stimulus phase and a response phase. In the stimulus phase, the subject made a saccade from firation mark $A$ to fination mark $B$. Sometime near or during the saccade, the compater genernted a brief stimules Inoh, condatiog of the letter 'I." After the sabject's eye had rested at the right-hand fintion mark for 100 msec, a cursor (consisting of a small dot) appeared there. This indicated the beginning of the response phase. By adfating a potentiometer knob, the subject moved the cursor until it was in the position where the subject thought the fiash had occurred. He or she looked at the cursor, and pressed a button to indicate he or she was doing so. This method of indicating the percelved stimulus position allowed a supplementary calibrition check to be made near the subject's response position; the computer verffied that dot position and measured eye position corresponded to better than 1/2 deg, otherwise rejected the response.

lie on the saccade-like contour showing the progress across the display of the particular retinal location stimulated. Errors in flash localization appear as deviations of the data points from these contours. The contours were obtained by measuring typical saccades of the three subjects during the experiment. The data representation is the same as that used by
Bischof and Kramer, and is explained further in Figure 2.

Accuracy before and after saccade. The main result to observe for flashes occurring before or after (but not during) the saccade is that the extent to which responses are veridical depends on the retinal location stimulated. For foveal and near foveal flashes ( 0 and $\pm 2.4 \mathrm{deg}$ from fovea), data points before and after the saccade lie near the contours indicating veridical response. For more peripheral flashes $( \pm 4.8$ and $\pm 7.2 \mathrm{deg}$ from fovea), data points show greater variability. Their mean position is also systematically displaced with respect to the veridical. This is more clearly seen from the filled circles in Figure 6, which plot median location error as a function of retinal eccentricity instead of time. The upper figures pool data in Figures 3, 4, and 5 over all times before the

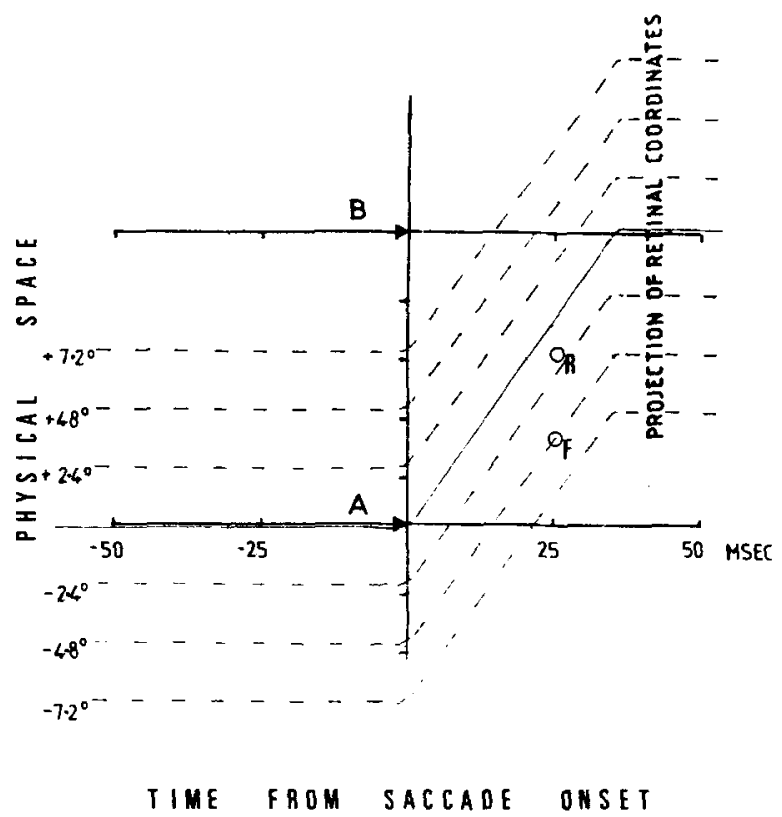

Figare 2. Coordinate system used to display the results. The ordininte AB is position on the display; the abectasa is time with respect to saccade onset. $A$ and $B$ are the white triangalar fixathon marks continnously vidble on the sereen. The saceade-shaped lines show where certain retinal projections are projeeted on the display sereen as time advances: the solid saccade-shaped line shows the progreas of the foven, which moved from $A$ to $B$, and the dotted saceade-shaped lines show the progress of the stx other retinal locntions that the fish can stimubte in the experiment. For example, point $F$ on the dotted curve marked -4.8 indicates the position on the display screen of the projection of retinal location -4.8 at instant 25 msec after saccade onset: 3.6 deg right of fintion mark A. To understand how data points will be plotied, asome that a fosh oceurred at this position and time. If the rabject made no error in his estimation of finh location, a data point would be plotted at F. If the subject made an error and anw the fash, for example, $7.2 \mathrm{deg}$ to the right of $A$, then the data point would be plotted at $\mathbf{R}$. In general, if the subject made no errors at all, all his data points for nashes impinging on retinal location -4.8 would lie ou the dotted curve marked -4.8 . 

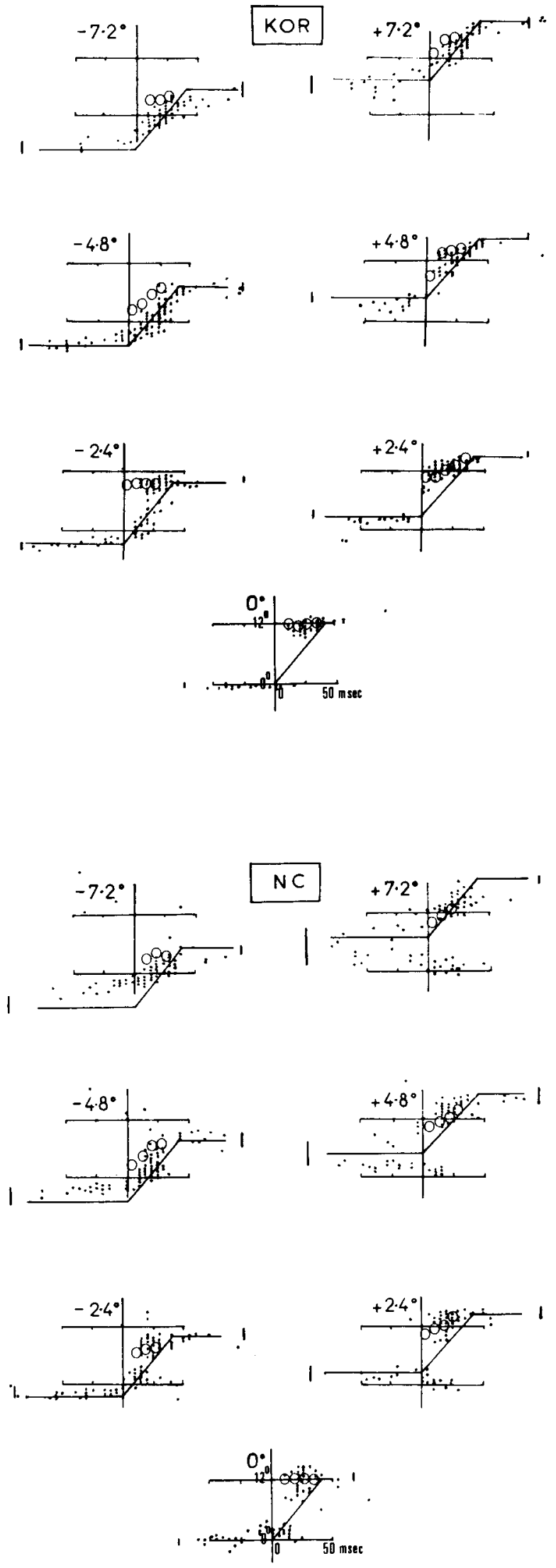
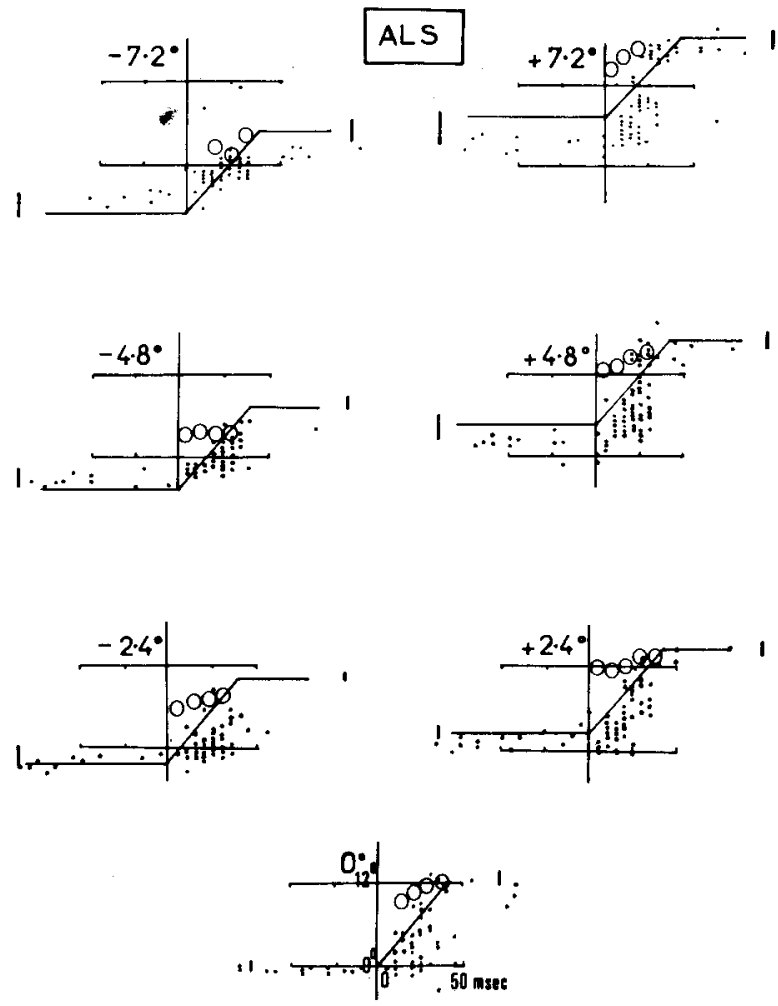

Figures 3, 4, and 5. Results of the saccade experiment for SubJeets K.O.R., N.C., and A.L.S. Each point is one response. Coordinate system used is that shown in Figure 2. A different graph is plotted for each retinal location from -7.2 to +7.2 deg stimulated by the flash. In each graph, the saccade-like contour shows the trajectory of the projection of the considered retinal location across the display. If responses were correct, they should lie on these trajectories. Responses to flashes oceurring either before or after the saccade correspond to data points that lie either left or right but not within the region of the dingonal line indicating the period during which the eye was making a sacende. Responses to fiashes occurring during the saccade lie within this saccadic region. The vertical bars are the ranges of mislocation errors obtained in the no-saceade experiment. The bar on the left of a given graph indicates the range of errors made in the no-saccade experment when the eye was stationary, fixating mark A, and a flash occurred at the retinal location corresponding to that graph. The bar on the right indicates the range of errors made when the eye was fxating mark $B$ and a fash occurred at that same retinal location. The open circles are median results obtained in the movingscene experiment. Each circle corresponds to five responses. 
saccade; the lower figures pool over all times after the saccade. In all cases, there is a definite dependence of location errors on retinal location stimulated. In four cases, this dependence consists in an underestimation of the veridical eccentricity of the flash (cf. negative slope of the curves). In the two other cases (K.O.R. and A.L.S., lower graphs), a different pattern exists, but there is still a clear variation of the effect with retinal location stimulated.

Accuracy during the saccade. Accuracy in estimating flash position during the saccade was variable across subjects. Nevertheless, here also, when the data for each subject are considered, there are definite differences for the different retinal locations stimulated by the flash. Thus, for Subject K.O.R., foveal flashes were localized at the fovea's departure point at $A$, or at its arrival point at $B$, but never near their veridical position in between. However, for nonfoveal flashes, Subject K.O.R.'s data show smoother curves and the responses are much closer to veridical. The same differences between foveal and peripheral flashes can be seen for Subject N.C., except that for positive retinal locations there was a tendency to see flashes at fixation marks A or B rather than in their veridical positions. Subject A.L.S.'s data are much noisier. They can be summarized by saying that for negative retinal locations she frequently saw flashes near fixation mark $\mathrm{A}$, whereas for positive and foveal retinal locations the curves were closer to veridical.

\section{Retinal Explanations}

The data strongly confirm Bischof and Kramer's finding of a retinal-locus effect. ${ }^{1}$ However, this does not mean that their interpretation in terms of a "coordinate system" version of the extraretinal signal is correct. Is there an explanation in terms of retinal rather than extraretinal mechanisms? Two retinal mechanisms may be at work in generating the data. (Note that by "retinal" we mean mechanisms brought into action by light falling on the retina, even though they may have a nonretinal component such as response bias. Mechanisms of "extraretinal" origin are related to the position of the eye in the orbit and are not mediated by information on the retina.)

Peripheral/foveal differences in the visual system. The retina is not homogeneous. Peripheral vision differs from central vision in a variety of ways: it has poorer acuity and poorer contrast sensitivity, there are rod-cone differences and differences in persistence, etc. These factors, which have nothing to do with the movement of the eye, may partially explain the systematic dependence of Bischof and Kramer's results on the retinal location stimulated by the flash. Indeed, it is known that even for the stationary eye, when a brief flash impinges on noncentral parts of the retina, systematic errors are made in estimating the spatial position of a flash. This is true in the dark (Osaka, 1977) and in the presence of a visible back- ground (Leibowitz, Myers, \& Grant, 1955; see also Mateeff, Mitrani, \& Yakimoff, 1977a, 1977b). In general, the work shows an underestimation of peripherally viewed distances. ${ }^{2}$ Other work (e.g., Newsome, 1972; Helmholtz, 1909/1925) shows systematic underestimation of the size of peripherally presented objects.

Background spearing. As pointed out by L. Matin (1976, referring to unpublished work of Matin, Matin, Bowen, \& Kowal, 1969), by Shebilske (1977, p. 31), by MacKay $(1970,1973)$, and by L. Matin, Stevens, and Picoult (1983), the fact that the Bischof and Kramer experiments were done in conditions of normal illumination and not in the dark means that information was available on the retina during the saccade which the subjects might have used to make their judgments, instead of relying on information from extraretinal sources. The most obvious hypothesis would be that at the moment of flash occurrence, the subject notes the instantaneous position of the flash relative to nearby reference points (in the present case, fixation marks $A$ and B), and uses this estimation to make his response afterwards. If the subject can do this accurately, his position judgments will be accurate. The fact that there are systematic errors in flash location can be attributed to periphery/ fovea differences, but it also may be related to smearing of the retinal image of the background caused by the eye movement. Localization of a brief flash in the presence of background smearing may give rise to complex retinal events leading to systematic biases in localization. This may be a further source of error for that subset of the data in the Bischof and Kramer and Mateeff paradigm that corresponds to cases when the flash is presented while the eye is moving.

\section{NO-SACCADE EXPERIMENT}

This experiment determines the role played in the saccade experiment by the first kind of retinal effect, namely, properties of the visual system leading to foveal/peripheral differences in localization ability. The influence of extraretinal information that may be present in the saccade experiment is removed by maintaining the eye fixed. Comparison with the saccade experiment is limited to cases in that experiment in which there is no additional effect of retinal smearing, that is, to cases in which the flash is presented before or after the saccade, at which time the eye is stationary.

\footnotetext{
Method

Subjects K.O.R., N.C., and A.L.S. were retested under exactly the same conditions as the saccade experiment, except that the subject never made a saccade. Instead, during the stimulus phase of the experiment, the subject continuously looked at either fixation mark $A$ or fixation mark B, depending on experimental condition. Two hundred milliseconds after the computer detected
} 
accurate fixation, a flash occurred at a position on the screen chosen by the computer so as to impinge upon one of several retinal locations, as in the saccade experiment. Since the eye was fixating, these corresponded to fixed locations on the screen, at least to within the accuracy determined by the subject's fixation accuracy and the accuracy of the calibration (1/3-1/2 deg). The interval of $200 \mathrm{msec}$ was chosen so as to approximately simulate the conditions in the saccade experiment, in which the flash appeared at an instant somewhere around saccade onset, that is, about one saccadic latency (ca. $200 \mathrm{msec}$ ) after an accurate fixation at fixation mark $A$ was detected. The response phase of the experiment was identical to that of the saccade experiment.

\section{Results}

The open circles in Figure 6 plot mean errors made by the subjects as a function of retinal location stimulated. For comparison, the errors made in the saccade experiment before and after the saccade are shown as filled circles. The trends of the two sets of curves are similar. The absolute value of the errors in the no-saccade experiment are smaller or equal to those in the saccade experiment. This shows that a sizable portion and sometimes all of the errors made in the saccade experiment, in cases before the eye starts moving or after it has stopped moving, can be explained by the no-saccade data, that is, by assuming that the position sense of the peripheral retina gives systematic errors. (It is interesting to note that the errors are different, depending on whether the subject is fixating mark A or mark B. This shows a dependence of errors on the visual background.)

The fact that the errors in the saccade experiment are sometimes larger than what would be predicted from the no-saccade experiment shows that there may sometimes be an additional source of error in the saccade experiment. However, this extra error is comparatively small. It could have an extraretinal source or it could be caused by that portion of the responses which were sufficiently close to saccade onset or offset to be affected by the retinal mechanisms of background smearing to be discussed later.

\section{MOVING-SCENE EXPERIMENT}

This experiment determines the role played in the saccade experiment by the second kind of retinal effect postulated above, namely background smearing. By moving the background rather than the eye, the experiment simulates the retinal disturbances caused by eye movement, but without the involvement of a possible extraretinal signal. The experiment is similar to that of MacKay (1970). However, Mackay did not disconfound retinal position stimulated and moment of stimulation, so comparison of his results with those of Bischof and Kramer and the present saccade experiment is not possible. This is remedied here by measuring separate time-error curves for each retinal location stimulated by the flash.

\section{Method}

A mechanical apparatus designed to model the conditions of the saccade experiment was constructed. A black slide, of the same width as the display screen of the saccade experiment, moved behind a stationary edge with a mark in the middle that served as fixation point to be continuously fixated by the subject. The black side was attached to the apparatus frame by an elastic band so that, when released from a position on the right, it would move in about $\mathbf{4 0} \mathrm{msec}$ to a resting position on the left. The black slide carried two white triangles corresponding to the fixation marks in the saccade experiment. The subject started with the slide so that triangle $A$ was positioned above his fixation mark on the stationary edge. While the subject maintained his eye steady on this mark, the experimenter released the slide to allow it to move rapidly to a position that brought triangle $B$ to a point that coincided with the subject's fixation mark. The subject held his regard steady throughout. Holes in the slide and in its frame could be opened so that, at a chosen moment during the slide's movement, a brief flash was projected at a chosen position on the subject's retina. Light for the flash came from a light bulb behind the apparatus. The retinal locations used were the same as in the previous experiment, but fewer flashes were used. It was assumed, as an approximation, that the slide moved at constant speed above the fixation point. Flashes could be delivered at instants corresponding to $1 / 10,3 / 10,5 / 10,7 / 10$, and $9 / 10$ of the saccade path. As in the saccade experiment, ambient lighting was kept the same during experimentation and was adjusted so that the flash could be easily detectable at the greatest retinal eccentricities.

The subject's task was to point to the location where he saw the flash. In this analog of the saccade experiment, the slide models the display screen and the stationary fixation mark corresponds to the fovea. Therefore, to make the task comparable to the saccade experiment, the subject made his location judgment relative to the moving slide and not relative to his stationary fixation point.

\section{Results}

For comparison with the saccade experiment, the results of the moving-scene experiment are plotted as open circles on the corresponding graphs of the saccade experiment (Figures 3, 4, and 5). This is justified because, as far as relative motion is concerned, the figures can still be considered to show the progress of the retina across the display, even though now the display is moving rather than the retina. Each open circle is centered on the median of five responses given by the subject. The dispersion of the responses was very small, and no larger than the diameter of the open circles.

The most important characteristic of the movingscene data was also observed in the saccade experiment: there are different patterns of error for the different retinal locations stimulated by the flash. As in the saccade data, foveal error curves differ from peripheral ones in the sense that they show little (Subject A.L.S.) or no (Subjects K.O.R. and N.C.) variation with time of flash. Foveal flashes are localized as though the retina "carried with it" the excitation caused by the flash, and assigned it to the position in space corresponding to the eye's final position (cf. K.O.R. and N.C. retinal location 0, in particular). Data for nonfoveal flashes also are similar to those for the saccade experiment. Nonfoveal flashes are not simply localized as though the retina carried with 

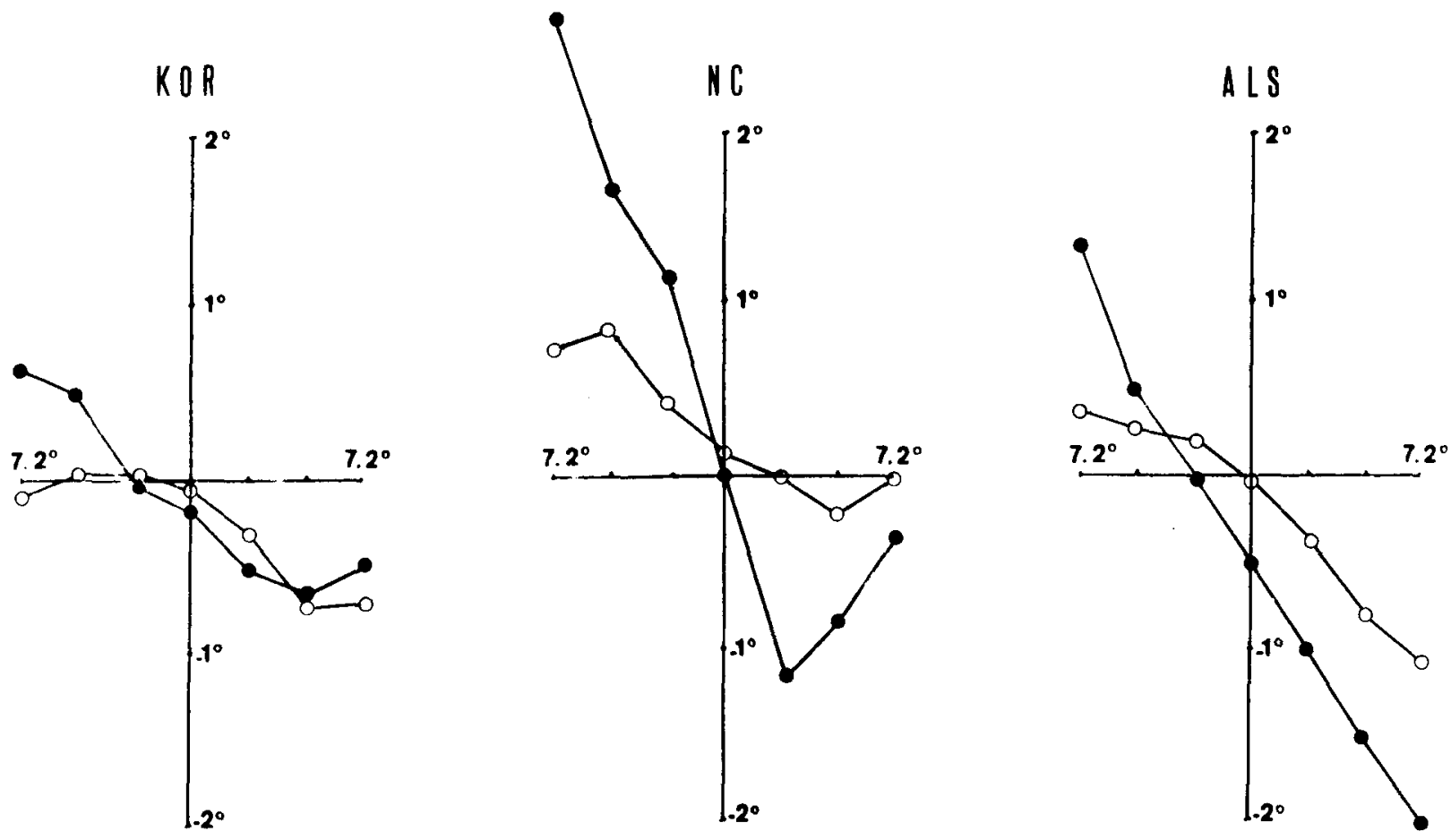

\section{Errors made before saccade}
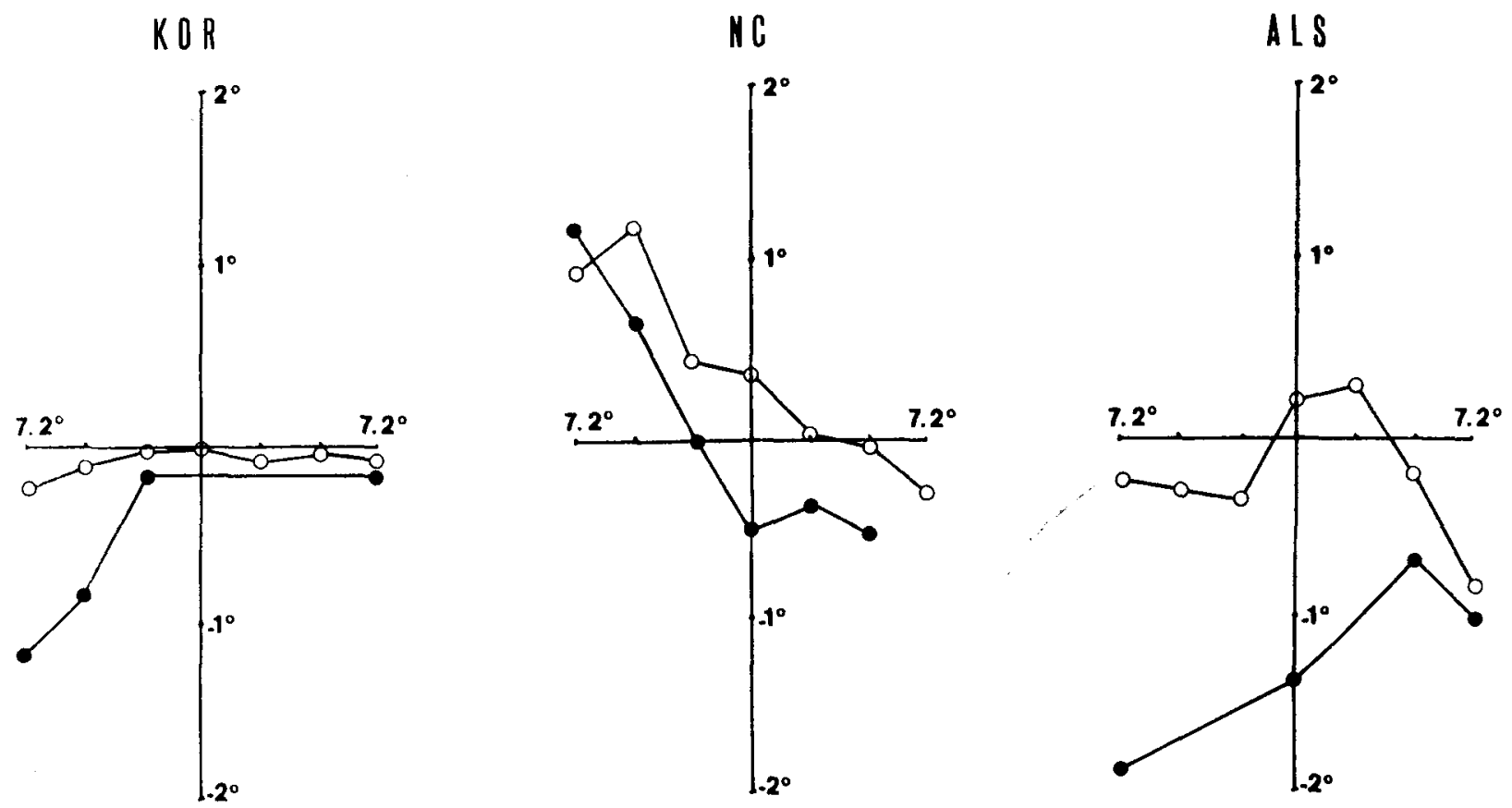

\section{Errors made aller saccade}

Figure 6. Filled circles: Subset of the results of the saccade experiment for cases in which the flash occurred before (upper curves) or after (lower curves) the saccade. Median location errors are plotted as a function of retinal location stimulated. Open circles: Mean location errors for the no-saccade experiment. 
it the excitation caused by the flash: estimated flash eccentricity lies in between what would be predicted from this hypothesis and the veridical position shown by the saccade-like contours in the graphs. Furthermore, the estimated flash position is not constant in time as would be predicted if localization took place as a function of the retinal stimulation remaining after the apparatus came to rest. Rather, estimated flash position varies with the moment of occurrence of the flash during the slide movement.

\section{Discussion}

Since the eye is stationary here, the fact that strong mislocation errors are found cannot be related to the growth of an extraretinal signal. ${ }^{3}$ Despite differences in experimental apparatus and procedure, the pattern of errors has the same qualitative and in many cases quantitative characteristics as the portion of the errors in the saccade experiment that occur for withinsaccade flashes.

\section{INTERMITTENT-FLASH EXPERIMENT}

The above experiments strongly suggest that a large part, if not all, of the location errors observed in the saccade and Bischof and Kramer experiments may have a retinal rather than an extraretinal cause. However, some extraretinal effects may still be present, and the present experiment is an attempt to measure them.

If the mislocation effects observed in the saccade experiment were due to the action of an extraretinal signal, then over a period of time, which may be long or short and synchronous or not with the saccade, the signal must make a change of about the same magnitude as the saccade amplitude. This predicts that if the same retinal position is stimulated several times intermittently during the period of growth of the extraretinal signal, then the perceived impression should be one of several flashes physically separated in space by about a distance corresponding to the saccade amplitude.

\begin{abstract}
Method
The experiment involved the same subjects and conditions as the saccade experiment, except that instead of the single flash projected on a given retinal location in each trial, seven flashes were projected by the computer at consecutive 5 -msec intervals, all impinging on that same retinal location. This was, of course, possible only to the extent to which the computer was able to accurately track and project flashes on a given retinal position during the saccade. The calibration accuracy of the apparatus was verified, at the initial fixation point and at the position of response, to be no more than $1 / 3$ and $1 / 2 \mathrm{deg}$, respectively. Possible flash positions and the instants of occurrence of the first flash in the series of seven flashes were the same as in the saccade experiment.
\end{abstract}

\section{Results and Discussion}

In all trials, the subjects perceived a small clump of flashes in which the successive flashes were super- imposed to a greater or lesser extent. Of interest was the maximum spread of the flashes seen, and only this was recorded for each subject. It was $1.3 \mathrm{deg}$ for K.O.R. and $2 \mathrm{deg}$ for N.C. and A.L.S.

Since $2 \mathrm{deg}$ is $1 / 6$ th of the total 12-deg saccade, this result shows that the extraretinal signal grows no more than 1/6th of the total saccade extent in the temporal vicinity of the saccade. In fact, the small spread that is found is wholly attributable to calibration error, inasmuch as a 2-deg error is of the same order as that expected through calibration error from a series of seven flashes that each can be about $1 / 3$ to $1 / 2$ deg away from the true retinal location to be stimulated.

A second experimental condition was run in which the series of seven flashes was always delivered starting at only one critical moment, namely the moment the saccade was detected. Since the greatest changes in the graphs of mislocation errors occur just after saccade onset in Mateeff's (1978), Bischof and Kramer's (1968), and the present saccade data, this is also where a calculated extraretinal signal will be changing the fastest. In consequence, this should be the moment at which the intermittent flashes should be most spread out. However, as before, for all retinal positions used in the saccade experiment, no spread larger than $2 \mathrm{deg}$ was reported. It must be concluded that any extraretinal signal cannot have changed by more than 1/6th of the total saccade extent between saccade onset and the seventh flash, $35 \mathrm{msec}$ (or about the saccade duration) later. ${ }^{4}$

L. Matin, E. Matin, and Pola (1968, unpublished work cited in L. Matin, 1976, p. 206) have performed an experiment whose results concord with those of the present experiment. If two flashes are flashed successively during the saccade with a brief interval between them, the perceived distance between the flashes reflects mainly their retinal offset, and not any change in the amplitude of an extraretinal signal.

\section{POSSIBLE RETINAL MECHANISMS}

The above three experiments show that in the data of Bischof and Kramer and of Mateeff, as well as that of the saccade experiment, the influence of an extraretinal signal, considered as a scalar or coordinate system of scalars, is minimal. The mislocation phenomena observed may, to a large extent, be explained in terms of retinal effects related to peripheral/ foveal differences in the visual system and/or to the presence and smearing of the background caused by the eye movement. The following sections present detailed information on mechanisms that show how these two types of retinal phenomena might produce the observed error patterns. It is assumed that the subject has no extraretinal information about eye position other than knowledge of the approximate amplitude and approximate moment of occurrence of the saccade. 


\section{Errors for Flashes Before and After the Saccade}

For flashes occurring when the eye is stationary before or after the saccade, the pattern of errors can be explained by appeal mainly to the first retinal mechanism, namely systematic differences between peripheral and foveal vision leading to errors in estimation of position. This was shown by the data in Figure 6.

It is interesting that this explanation in terms of periphery/fovea differences predicts that there will be no evolution of the size of location errors over time during the periods before and after the saccade. There is insufficient data in the present saccade experiment to be sure of this, but there is certainly no obvious systematic time evolution visible.

\section{Errors for Flashes During the Saccade}

What strategy might the subject adopt to localize a flash that occurs during a saccade? There are problems with the simple idea that he notes the relative position of the flash with respect to the background at the moment the flash occurs. The remanence of the visual system is considerable: the movement, caused by the saccade, of the visual scene over the retina, undoubtedly builds up to a complicated pattern of excitation whose rise and decay time is of the same order as the saccade's duration. When a flash occurs superimposed on this pattern at a particular retinal location, the excitation due to the flash itself also takes a certain time to build up and to decay. In order to estimate the position of the flash, a subject must choose some moment and some landmarks within the overall spatiotemporal pattern of excitation with respect to which to make his or her judgment. It seems most likely that the location of the flash should be decided on the basis of the final, cumulated pattern of stimulation and not on any instantaneous time-slice of stimulation. This is because, first, the visual system is not capable of distinguishing events closer together in time than the period of temporal integration, which, being at least $50 \mathrm{msec}$, is longer than the duration of the saccade itself, and, second, decision processes themselves probably cannot separate events like the appearance of the flash from immediately preceding and following retinal disturbances.

Consider the case of foveal flashes. The data of saccade and moving-scene experiments show that subjects tend to localize foveal flashes at the final fixation mark, B, as though the retina "carried with it" the excitation caused by the flash. There is no influence of the moment of occurrence of the flash. This is consistent with the idea that localization occurs only on the basis of the pattern of excitation on the retina after the saccade, since at this time the remanence from the flash coincides with fixation mark B.
The case of peripheral flashes is harder to explain because the data show a clear variation of mislocation errors with time of stimulation. There must be some differences between periphery and fovea that makes a time dependence appear. It we assume that decisions are based on the cumulated evidence after the saccade, then, in contrast to the case for foveal flashes, we must assume for peripheral flashes that there is some difference in the final pattern of stimulation as a function of their moment of occurrence. One possibility is that remanence may be shorter in periphery than in foveal vision, so that what is left of the stimulation after the saccade depends on how early in the saccade the flash occurred.

Little work exists in the literature on the subject of periphery/fovea remanence differences. However, the following facts, though not speaking directly to the issue, concur in suggesting that under some conditions a foveal excitation takes longer to decay than a parafoveal one. First, there is some data (e.g., Hartmann, Lachenmayr, \& Brettel, 1979) that suggests that, under certain conditions of lighting, flicker fusion frequency is higher in parafoveal regions. Incidental evidence to support this can be obtained by looking at a small TV screen. Under fairly bright lighting conditions, when seen in near peripheral vision, the TV image will appear to flicker slightly, but fixated foveally, no flicker will be seen. A second source of evidence comes from observations that can be made by waving a light in a dim room while the eye fixates a stationary point. The length of the streak made by the moving light is much shorter in peripheral vision than in foveal vision. A final observation, already mentioned by Helmholtz (1909/1925), is that afterimages remain visible longer in central vision.

Using this idea, one might suggest the following account for what happens when the flash impinges on a peripheral part of the retina. The point on the retina excited by the flash will preserve its activity only for a certain time, say $\mathrm{T}$ after its occurrence, where $T$ depends on the retinal location struck, and is presumably long for the fovea but short for periphery. At this moment, $T$, when the activity caused by the flash is about to disappear, the two white fixation triangles will already have moved through a certain distance, creating two streaks of excitation on the retina. The length and position of these streaks relative to the position of the fading flash excitation is all the subject has to go by in performing his localization task. Suppose that he chooses some intermediate position along the streaks as corresponding to the "true" triangle position at moment $\mathrm{T}$, and estimates the distance of the flash excitation from this intermediate point (in doing so he may make an error of estimation caused by inaccuracy in peripheral distance estimations). After the eye comes to rest, he measures off this distance from the appropriate tri- 
angle, and says he saw the flash at the resulting position.

The predictions made by this hypothesis are dependent on the dynamic characteristics of the smears caused on the retina by the eye movement and of the excitation caused by the flash. As shown by E. Matin, Clyner, and L. Matin's (1972) elegant experiment and by van der Wildt and Vrolijk's (1981) work on the asymmetrical propagation of inhibition (see also Long, 1982, and Burr, 1980), these will be complicated, and they will depend on the parts of the retina struck by the streaks and flash. Given the present lack of knowledge about the dynamic characteristics of different regions of the retina, it is difficult to make precise predictions. However, following the arguments given in the Appendix, two extreme cases can be isolated, which give the limits within which the responses should lie if the present hypotheses are correct. These cases are shown in Figure 7.

As is evident from a comparison with Figures 3, 4 , and 5 , the true data fall within the predicted limits. The extreme cases leave a lot of leeway for the true data, so it is not surprising to find agreement. However, one fundamental prediction arises from the basic concept inherent in the present discussion, namely, that localization occurs at time $T$ after flash occurrence, where $T$ is long in the fovea and short in the periphery. This prediction is that mislocation phenomena should depend on the speed of the eye movement, since the faster the eye moves, the farther it will have moved during time T. Pilot experiments done by changing the slide velocity in the movingscene apparatus support this prediction. Furthermore, MacKay (1970) found that mislocation errors

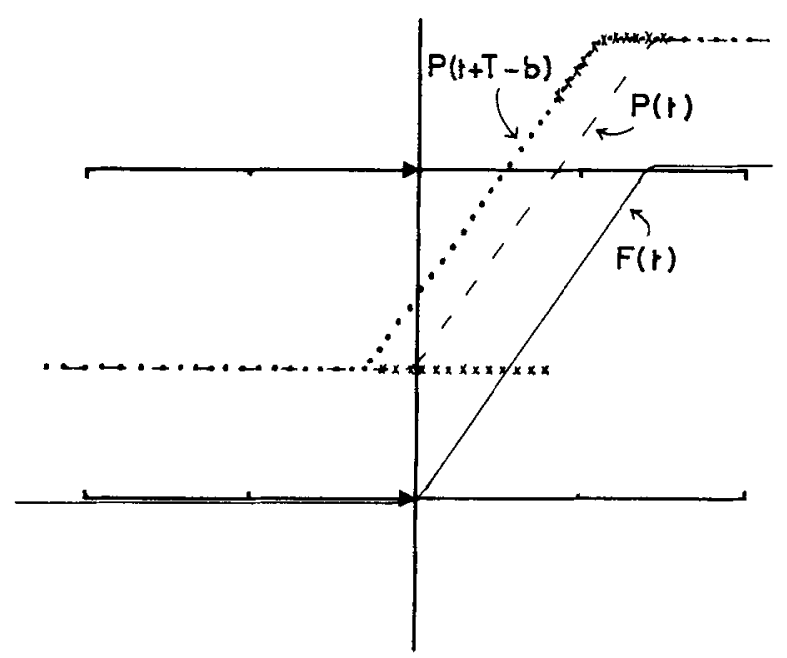

Figure 7. Theoretical predictions. $F(t)$ is the trajectory of the foved projection across the display, $P(t)$ is that of a given peripheral location. A flash is assumed to fall on this peripheral location. The dotted contour labeled $P(t+T-b)$ shows the positions where flashes would be seen under one extreme model; the crosses correspond to the other extreme model discussed in the Appendix. doubled when the velocity of the simulated saccade was multipled by 4.5 .

\section{GENERAL DISCUSSION}

The starting point for the present study was the observation that most work on the mislocation phenomenon has neglected to take into account the study by Bischof and Kramer (1968) showing that the time course of mislocation errors may be different when the flash impinges on different retinal locations.

The present series of experiments reexamined Bischof and Kramer's claim. The saccade experiment confirmed that the retinal-location effect does exist. However, the subsequent series of three experiments showed that a significant portion of the effects can be attributed to: (1) differences in the visual system's response to foveal versus peripheral flashes (different sensitivity to position, possibly differences in temporal response); and (2) complicated retinal events caused by movement of the visual scene across the retina. It is thus not necessary to generalize the notion of extraretinal signal to a "moving coordinate system" in the way Bischof and Kramer suggested. More important, it is not necessary to use the extraretinal signal notion at all: direct measurement of the change in extraretinal signal during the saccade shows the change, if it exists at all, to be less than 1/6th of the size of the saccade.

To what extent can the extraretinal signal also be dispensed with in explaining the mislocation effects found by other authors?

The work of Mateeff (1978) was done under conditions of visible background, just as were the present experiments. The same retinal mechanisms may therefore have been active in Mateeff's experiments and the present ones. Unfortunately, direct comparison of the data from the two experiments is not possible, because Mateeff failed to disconfound time of stimulation and retinal location stimulated. However, a limited comparison is possible if the saccade experiment data is purposefully confounded by collapsing over all retinal locations. This was done in Figure 8. Data will be similar to Mateeff's experiment only if the same combination of retinal locations are stimulated. There is no guarantee that this was the case. Nevertheless, the data are similar to Mateeff's, particularly those for Subject K.O.R., where the resemblance is striking. For Subjects A.L.S. and N.C., the similarity is less good, but the range of errors is similar to that observed by Mateeff, one of whose subjects also had a negative error before saccade onset. There is therefore a strong suggestion that the extraretinal component in his data was minimal and that the phenomenon observed by Mateeff was the same as that observed here.

A further point concerns location errors before and after the saccade. In Figure 8 and in Mateeff's 


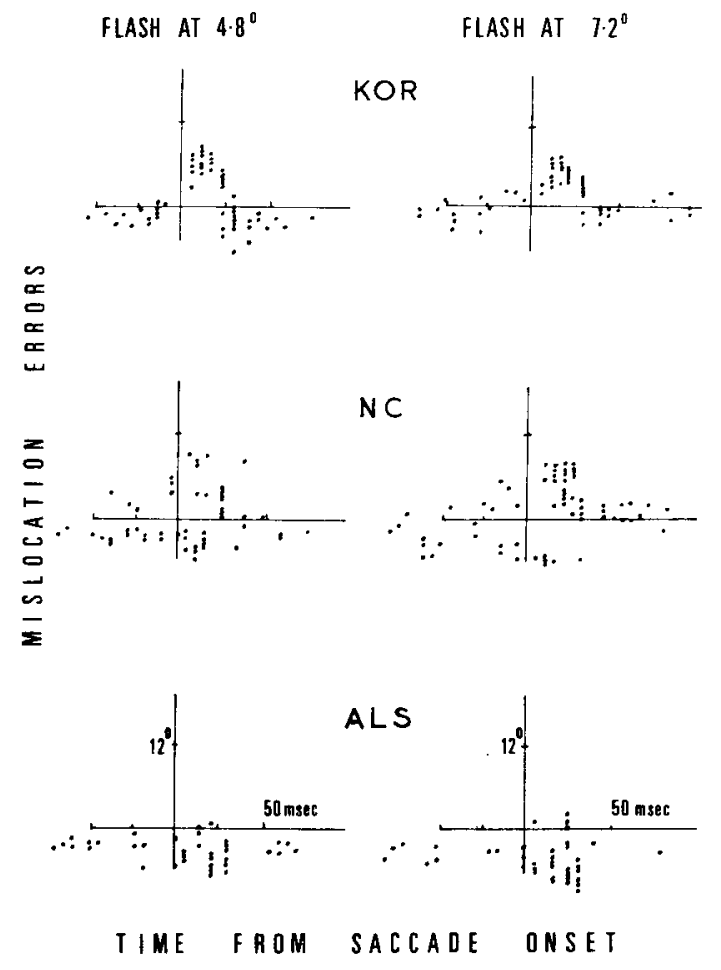

Figure 8. Results of the saccade experiment pooled over all retinal locations, but keeping flash position in space constant. For a comparison with Mateeff (1978), mislocation errors are plotted instead of absolute estimated nash position. If there were no errors, all points would lie on the $x$-axis. Two flash positions in space for the flash were considered, one on either side of the midpoint between $A$ and $B: A+4.8 \mathrm{deg}$ and $A+7.2 \mathrm{deg}$ (i.e., $1.2 \mathrm{deg}$ on either side of the midpoint between $A$ and $B$ ).

data, these errors are not zero and they show changes as a function of time. Some workers have taken this to be evidence that the mislocation effect has an extraretinal origin, the argument being that retinal effects cannot be active before and after the saccade, since the eye is stationary. In fact, however, the apparent deviations from zero and the time evolutions, at least in the saccade data, are an artifact of the way the data are pooled. In Figure 8 and in Mateeff (1978), data are pooled over an uncontrolled variety of retinal locations, each location giving rise to different systematic errors. The resulting mean error therefore mainly reflects differences in the distributions of retinal locations sampled at different times. A final point concerning Mateeff's notion of "generalized moment of stimulus presentation" is given in the Appendix.

The work of L. Matin and Pearce (1965), L. Matin, E. Matin, and Pearce (1969), L. Matin, E. Matin, and Pola (1970), and Monahan (1972) was done in the dark, so there was no smear created on the retina by a moving background. However, differences between the responses of the visual system to peripheral and foveal flashes undoubtedly still exist. It is inter- esting to speculate whether such differences might account for the data these workers obtained.

Suppose no extraretinal information is available to subjects other than the knowledge of the approximate moment that the saccade occurred, and the approximate size of the saccade. Suppose, as before, that subjects make their judgments only on the basis of the information available on the retina after the saccade. Since, after the saccade, the eye is resting in the dark, and since the initial fixation point disappeared more than $300-400 \mathrm{msec}$ before the saccade, the only retinal information available is the remanence of the test flash. Now, the strength of this remanence is an indication of the moment at which the flash occurred, with some inaccuracy introduced by periphery/fovea differences. If the remanence was very weak, the subject might assume that the flash had occurred fairly near the beginning of the saccade, that is, before the eye had got very far towards the saccadic target. In that case, if the remanence was on the left of the fovea, he would guess that the flash had occurred to the left of the initial fixation point. Similarly, if the remanence was on the right, the subject would guess that the flash had occurred to the right of the initial fixation point. On the other hand, a fairly strong remanence would suggest that the flash had occurred late in the saccade. Its position relative to the fovea at the arrival point is therefore quite near its veridical position. Knowing approximately the amplitude of the saccade, the subject can estimate whether this position is left or right of the initial fixation point.

Use of such a strategy will lead to a pattern of leftright judgments in which the point of subjective equality begins to move slowly some time before the saccade and reaches its final stable position only well after the saccade. This slowness of the change in the point of subjective equality (and therefore of the deduced extraretinal signal) will be seen because the subject has only a poor conception of the exact time at which his saccade occurred, and so he attempts (inappropriately) to use the remanence strategy for flashes occurring both before and after the saccade.

The above discussion shows that even in the dark, in the conditions of Matin and his co-workers' experiments, there may be ways of explaining the systematic time dependence of subjects' judgments without making use of the notion of a time-varying extraretinal signal.

In conclusion, the present experiments show that when there is a visible background, flash mislocation data can to a large extent be explained by retinal mechanisms. The action of these retinal mechanisms can be observed only if data are plotted separately for the different retinal locations stimulated by the flash. One of these retinal mechanisms, namely the difference between the peripheral and foveal position sense, may be active even under conditions of stim- 
ulation in which there is no visible background. A significant body of research done in the dark has not considered this possibility. The influence of the extraretinal signal on mislocation errors in the dark therefore has yet to be determined.

\section{NOTES}

1. The present data supplement the findings of Bischof and Kramer (1968) by a more systematic exploration of retinal locations and instants of flash occurrence. In particular, in the present experiment, flashes could occur beyond the saccade endpoints, whereas in Bischof and Kramer's experiment flashes always occurred between the two fixation marks. For retinal regions and flash instants common to both studies, very good agreement is found in the data. Thus, the data for Bischof and Kramer's Subject $\mathrm{K}$, for whom there were data for retinal locations -7 to +2 scale marks (corresponding to -3.5 to +1 fifths of the saccade) is very similar to that for the present Subject K.O.R. for retinal locations -7.2 to $+2.4 \mathrm{deg}$, or -3 to +1 fifths of the saccade. (My further experiments showed that the mislocation errors become larger when the saccade becomes larger. For the purpose of comparison with other studies, it therefore appears preferable to measure the errors as a fraction of total saccade length.) Bischof and Kramer's Subject $\mathbf{N}$ is also very similar to my Subject N.C. as far as their data permit comparison; that is, their retinal locations -3 to +2 scale marks, or -1.5 to +2 fifths of the saccade, correspond approximately to our retinal locations of -2.4 to $+4.8 \mathrm{deg}$. It is important to note that this good agreement between the results of the two studies shows that the effects do not depend critically upon lighting conditions, flash remanence, visual environment, and saccade size (when errors are measured as a proportion of the saccade), all of which were different in the Bischof and Kramer study.

2. In experiments being done in my laboratory, the errors appear to consist of an underestimation of distances by about $10 \%$ in peripheral vision; that is, flashes appearing near fixed reference points tend to be located $10 \%$ nearer those points than they really are.

3. A possible counterargument to this might be the following: It might be argued that the growth of the extraretinal signal corresponds to an attentional shift accompanying eye movements, and a similar attentional shift could occur in the moving-scene experiment accompanying the slide movement. However, it must be noted that for the moving-scene experiment in the case of Subjects N.C. and A.L.S., it was the experimenter who determined the instant at which the simulated saccade occurred, whereas it was the subject himself who did so in the case of K.O.R. It would be very unlikely that the time course of an attention shift would be the same under conditions when the simulated saccade instant was determined by the subject and when it was determined by the experimenter. And yet comparison of the resulting graphs shows that the time courses of the mislocation effects are identical (cf., in particular, K.O.R. and N.C.). It can therefore be assumed that the mislocation effects found here are truly caused by retinal disturbances related to the image shift. The mislocation errors in the saccade experiment for flashes occurring during the saccade can therefore probably also be accounted for on the basis of purely retinal effects.

4. Although the straightforward version of the extraretinal signal hypothesis obviously cannot be reconciled with this result, at first sight it might be thought that Mateeff's "generalized moment of stimulus presentation" idea (cf. Appendix) could be slightly modified to account for the present data. It might be argued that the 5-msec interval between successive flashes was so short that the series of seven flashes would be considered by the visual system as just a single, long-duration flash falling on the given retinal location, to which a single "generalized moment" would be attributed. But note that the subjects did not perceive a single flash, but rather a small clump of flashes (on the screen, some flashes were sometimes seen as quite separate from others, though the total spread was never more than $2 \mathrm{deg}$ ). Under the retinal smear hypothesis, this is easily understood as being because calibration error caused each flash to land on a slightly different retinal location. Under Mateeff's theory, it would be necessary to suppose that the whole clump of flashes should be attributed to a single "generalized moment," even though they are neither temporally nor spatially contiguous. This seems to be stretching the theory too far. 5. I am indebted to J. Roufs for pointing this out to me.

\section{REFERENCES}

Bischof, N., \& Kramer, E. (1968). Untersuchungen und Überlegungen zur Richtungswahrnehmung bei Willkürlichen sakkadischen Augenbewegungen. Psychologische Forschung, 32, 185-218.

BUrR, D. (1980). Motion smear. Nature, 284, 164-165.

Hartmann, E., Lachenmayr, B., \& BretTel, H. (1979). The peripheral critical flicker frequency. Vision Research, 19, 1019-1023.

Hazelhoff, F. F., \& Wiersma, H. (1924). Die Wahrnehmungszeit. I. Die Bestimmung der Schnelligkeit des Wahrnehmens von Lichtreizen nach der Lokalisationsmethode. Zeitschrift für Psychologie, 96, 171-188.

Helmholtz, H. von. (1925). [Physiological optics] (Vol. 3; J. P. C. Southall, Trans.). Rochester, NY: Optical Society of America. (Original work published 1909).

Leibowitz, H. W., Myers, N. A., \& Grant, D. A. (1955). Frequency of seeing and radial localization of single and multiple visual stimuli. Journal of Experimental Psychology, 50, 369-373.

LoNG, G. M. (1982). Receptor interactions and visual persistence. Vision Research, 22, 1285-1292.

MacKAy, D. M. (1970). Mislocation of test flashes during saccadic image displacements. Nature (London), 227, 731-733.

MACKAY, D. M. (1973). Visual stability and voluntary eye movements. In R. Jung (Ed.), Handbook of sensory physiology (Vol. VII/3A, pp. 307-331). Berlin: Springer Verlag.

MATEEFF, S. (1978). Saccadic eye movements and localization of visual stimuli. Perception \& Psychophysics, 24, 215-224.

Mateeff, S., Mitrani, L., \& Yakimoff, N. (1977a). Localization of disappearance of a light target during tracking eye movements. I. Acta Physiologica et Pharmacologica Bulgarica, 3, 21-27.

Mateeff, S., Mitranı, L., \& Yak1moff, N. (1977b). Localization of disappearance of a light target during tracking eye movements. II. Acta Physiologica et Pharmacologica Bulgarica, 3, 62-67.

Matin, E., Clymer, A. B., \& Matin, L. (1972). Metacontrast and saccadic suppression. Science, 178, 179-182.

Matin, L. (1972). Eye movements and perceived visual direction. In D. Jameson \& L. M. Hurvich (Eds.), Handbook of sensory physiology (Vol. VII/4, pp. 331-380). Visual psychophysics. Berlin: Springer Verlag.

Matin, L. (1976). Saccades and extraretinal signal for visual direction. In R. A. Monty \& J. W. Senders (Eds.), Eye movements and psychological processes (pp. 205-219). Hillsdale, NJ: Erlbaum

Matin, L. (1982). Visual localization and eye movements. In A. Wertheim, W. A. Wagenaar, \& H. Leibowitz (Eds.), Tutorials on motion perception. New York: Plenum.

Matin, L., Matin, E., \& Peance, D. G. (1969). Visual perception of direction when voluntary saccades occur. I. Relation of visual direction of a fixation target extinguished before a saccade to a flash presented during the saccade. Perception \& Psychophysics, 5, 65-79.

Matin, L., Matin, E., \& Pola, J. (1970). Visual perception of direction when voluntary saccades occur. II. Relation of visual direction of a fixation target extinguished before a saccade to a subsequent test flash presented before the saccade. Perception \& Psychophysics, 8, 9-14. 
Matin, L., \& Pearce, D. G. (1965). Visual perception of direction for stimuli flashed during voluntary saccadic eye movements. Science, 148, 1485-1488.

Matin, L., Stevens, J. K., \& Picoult, E. (1983). Perceptual consequences of experimental extraocular muscle paralysis. In A. Hein \& M. Jeannerod (Eds.), Spatially oriented behavior. New York: Springer Verlag.

Monahan, J. S. (1972). Extraretinal feedback and visual localization. Perception \& Psychophysics, 12, 349-353.

Newsome, L. R. (1972). Visual angle and apparent size of objects in peripheral vision. Perception \& Psychophysics, 12, 300-304.

O'REgAN, J. K. (1978). A new horizontal eye movement calibration method: Subject-controlled "smooth pursuit" and "zero drift." Behavior Research Methods \& Instrumentation, 10, 393-397.

Osaka, N. (1977). Effect of refraction on perceived locus of a target in peripheral visual field. Journal of Psychology, 95, 59-62.

SHEbilske, W. L. (1977). Visuomotor coordination in visual direction and position constancies. In W. Epstein (Ed.), Stability and constancy in visual perception: Mechanisms and processes. New York: Wiley.

Sherrington, C. S. (1918). Observations on the sensual role of the proprioceptive nerve-supply of the extrinsic ocular muscles. Brain, 41, 323-343.

van der Wildt, G. J., \& Vrolijk, P. C. (1981). Propagation of inhibition. Vision Research, 21, 1765-1771.

von Holst, E., \& Mittelste AdT, H. (1950). Das Reafferenzprinzip. Die Naturwissenschaften, 20, 464-476.

\section{APPENDIX}

\section{Predictions for Flashes During the Saccade Using the Hypothesis of Periphery/Fovea Remanence Difference}

Suppose $t$ is time from the beginning of the eye movement, and suppose $P(t)$ is the position of the retinal location stimulated, measured with respect to the left fixation triangle. For a flash occurring at time $t$, localization takes place at time $t+T$, at which moment the retinal location stimulated by the flash has moved to position $P(t+T)$, measured with respect to the left triangle. ( $T$ is the remanence of the retina at the location stimulated.)

The first of the two extreme cases to consider is the case when the subject makes his localization with respect to the most recent position on the retina stimulated by one or the other of the triangles, let us say the left one. If the excitation caused by the triangle moving across the retina builds up instantaneously, then the excitation will be in the same retinal location as the triangle itself, and so the subject will estimate the flash position to be $P(T+t)$ from it.

Now, assume it takes time $b$ for the moving triangle stimulation to build up (b will be a function of the retinal position currently being traversed by the triangle, but will presumably be small-on the order of milliseconds). At the moment of localization $t+T$, the left triangle will be distance $P(t+T)$ from the retinal location stimulated by the flash, but excitation will not yet have built up at this position. Excitation will only have built up from the triangle at a position where it was $b$ time units earlier, that is, at distance $P(t+T-b)$ from the retinal location stimulated by the flash. $P(t+T-b)$ will therefore be the estimated flash position. This is indicated by the dashed curve in Figure 7. Note that the curve depends on the values of $T$ and $b$. $T$ is a function of the retinal position struck by the flash, and is therefore not a function of time; $b$ is a function of the retinal position currently being traversed by the triangle $A$, and so is a function of time.

The second of the two extreme cases to consider is that in which, instead of making his localization with respect to the most recent ("freshest") end of the streak created by the triangles moving across the retina, the subject makes his localization with respect to the other end of the streak, that is, its least recent ("stalest") end. If there were no decay or inhibitory effects at all, the stalest end of the streak would be at the retinal position occupied by the triangle before the eye began moving, that is, for the left triangle, the fovea. Localizing the flash with respect to the left triangle therefore means localizing the flash with respect to the fovea. A flash stimulating a given retinal position will be seen as at a constant distance from the left triangle, independently of its moment of occurrence. This conclusion becomes untenable, however, when one considers what happens if the flash occurs very late in the eye movement. In that case, the left triangle has almost arrived at its final position in the left periphery, and the right triangle is coming to rest near the fovea. Suppose that the retinal location stimulated by the flash is a little ways into the right periphery. Then it is unlikely that the subject would use the excitation on the fovea caused by the left triangle as a reference, since the right triangle's excitation is more recent and closer to the position stimulated by the flash. It therefore seems reasonable to postulate that there is a critical moment at which the subject changes from using the foveal excitation remaining from the left triangle to using the most recent excitation created by the right triangle. The critical moment will depend on the proximity of the retinal location stimulated by the flash to one or the other of these reference streaks. The crosses in Figure 7 show the mislocation errors predicted for a hypothetical value of this critical moment.

\section{Relation to the Notion of "Perception Time"}

The mechanisms presented here appear to be related to the old idea of Hazelhoff and Wiersma (1924), according to which there is a "perception time" delay between the moment of the flash and the moment it is "perceived." However, the perception time idea runs into two kinds of difficulties. First, once the flash is "perceived," its position must be compared with some standard (e.g., the scale) in real space. This standard must also be "perceived," and this will also take a certain time. "Perception time" must therefore actually be taken to be the difference in the time needed to perceive the flash and the standard. ${ }^{5}$ Secondly, note that Mateeff, Mitrani, and Yakimoff $(1977 a, 1977 b)$ have recently looked more closely at the predictions made by the simpler form of the notion of perception time, using target tracking rather than a saccade. They show that if the notion is to be retained, different perception times must be postulated, depending on the brightness of the flash, its probability of occurrence, and the velocity of the tracking. On neither neurophysiological nor cognitive grounds is it easy to understand why perception time should depend on tracking velocity. Also, as pointed out by Mateeff et al., there is a discrepancy between perception times measured in saccade experiments and in tracking experiments. All these facts may be better explained not in terms of perception time, but in terms of the relative location of streaks on the retina, as suggested here. 


\section{Relation to Mateeff's “'Generalized Moment of Stimulus Presentation"}

Mateeff (1978) postulated an extraretinal signal to account for his mislocation effects, and calculated the time course of the signal that would be necessary for it to account for his data. The signal calculated in this way starts to rise about $50 \mathrm{msec}$ before saccade onset (corresponding to mislocation errors appearing for flashes $50 \mathrm{msec}$ before saccade onset) and reaches the value required for veridical perception within $20 \mathrm{msec}$ after saccade end. He then tested the validity of this calculated signal by replacing the brief $(0.5 \mathrm{msec})$ flash by one of longer duration $(9 \mathrm{msec})$ and asking subjects to estimate the positions of the endpoints of the smear that had been seen. He found that if the previously calculated extraretinal signal was used to deduce what would happen in the new "streak" experiment, the wrong predictions were made for the portion of the mislocation curves preceding saccade onset. Since the calculated extraretinal signal rises slowly well before saccade onset, the beginning and end of a 9-msec flash presented well before saccade onset should be attributed to different locations in space. Instead, they are seen as strictly coincident. Only for flashes presented after the saccade begins does a streak with separate endpoints appear. To get over this problem, Mateeff proposed that, for a stimulus occupying a given retinal location, the visual system uses the extraretinal signal to calculate flash position only once, and that this is done not at flash onset, or at its offset, but at some intermediate moment which he called the "generalized moment of stimulus presentation.",

Although this is a very ingenious method of accounting for the data, the retinal mechanisms proposed here do equally well. For pre- and postsaccadic flashes, it is clear that since the eye is stationary, no streak will be seen. For flashes during the saccade, the subject makes his response by comparing the position of the streak on the retina created by the flash with respect to the position of the streak on the retina created by the scale. Although the streak created by the flash was previously of negligible length, because of the flash's negligible duration, now a longer duration flash gives rise to a significant streak. The exact length of this streak will depend on the dynamic properties of the regions of the retina that it crosses. Presumably the subject will choose some intermediate position on the streak caused by the flash, in addition to choosing, as before, an intermediate position on the streak caused by the scale. This modifies the earlier predictions made for instantaneous flashes in exactly the same way as Mateeff's "generalized moment of perception"' hypothesis. It can be concluded that insofar as Mateeff's data is concerned, both Mateeff's model and the retinal mechanisms proposed here can deal with the data. Only the retinal mechanisms, however, deal satisfactorily with the dependency of mislocation errors on retinal location shown by Bischof and Kramer (1968) and the present saccade and moving-scene experiments. Only the retinal mechanisms explain why, in conditions in which the eye is stationary, similar mislocation phenomena can be generated by moving the scene (moving-scene experiment, and MacKay, 1970).

(Manuscript received May 13, 1983; revision accepted for publication April 13, 1984.) 